Revista Brasileira de Agricultura Irrigada v.10, nº.2, p. 507 - 514, 2016

ISSN 1982-7679 (On-line)

Fortaleza, CE, INOVAGRI - http://www.inovagri.org.br

DOI: $10.7127 /$ rbai.v10n200382

Protocolo 382.16 - 26/11/2015 Aprovado em 14/04/2016

\title{
SALINIDADE DA ÁGUA DO RIO CURU - CEARÁ NO PERÍODO DE ESTIAGEM
}

\author{
Vanessa Ohana Gomes Moreira ${ }^{1}$, Fernando Felipe Ferreyra Hernandez ${ }^{2}$, Edilaine da Silva \\ Marques ${ }^{3}$, Nayara Rochelli de Sousa Luna ${ }^{4}$
}

\section{RESUMO}

A Bacia do Vale do Curu localizada no Estado do Ceará possui expressiva importância no abastecimento de água para o consumo doméstico, indústrias, atividades pesqueiras e, principalmente, para irrigação de áreas agrícolas. O Rio Curu, principal rio da Bacia, recebe água poluída de diversos locais, dentre eles, comunidades, viveiros de camarão, entre outros, que pode salinizar a água do manancial, colocando a produção agrícola e os solos da região em risco, portanto, este trabalho teve como objetivo avaliar a qualidade da água de todo o percurso do rio, levando em consideração a presença de sais. Foram escolhidos, no período seco, 21 pontos de coleta desde a nascente até a foz do rio. Nas amostras de água foram analisadas a condutividade elétrica (CE), o $\mathrm{pH}$, as concentrações de cálcio $\left(\mathrm{Ca}^{2+}\right)$, magnésio $\left(\mathrm{Mg}^{2+}\right)$, potássio $\left(\mathrm{K}^{+}\right)$, sódio $\left(\mathrm{Na}^{+}\right)$, cloreto $\left(\mathrm{Cl}^{-}\right)$, bicarbonato $\left(\mathrm{HCO}_{3}{ }^{-}\right)$, carbonato $\left(\mathrm{CO}_{3}{ }^{2-}\right)$ e sulfato $\left(\mathrm{SO}_{4}{ }^{2-}\right)$. No que concerne à presença de sais, na maior parte do trecho do rio Curu, as águas se encontram com restrição moderada para utilização na irrigação. No mangue e na foz do rio, a concentração de sais foi bastante elevada, porém próximos a estes pontos não há presença de áreas agricultáveis que possam utilizar estas águas. Conclui-se que, em termos qualitativos, a água do Rio Curu possui restrição moderada para irrigação na maior parte da sua extensão, com exceção dos pontos no mangue e na foz do rio, que pela influência da maré, possui quantidades elevadas de sais.

Palavras-chave: Bacia hidrográfica. Irrigação. Poluição.

\section{WATER SALINITY OF THE CURU RIVER - CEARA, DURING DROUGHT PERIOD}

\footnotetext{
${ }^{1}$ Engenheira Agrônoma. Estudante de Mestrado em Solos e Nutrição de Plantas. Departamento de Solos Fortaleza- CE. Brasil. E-mail: van_ohana1@hotmail

${ }^{2}$ Professor Titular do Departamento de Solos

${ }^{3}$ Professora do Instituto Federal de Educação, Ciência e Tecnologia

ECE.FBraßibrtatetexail: ferrey@ufc.br

mail: edilainemarques38@yahoo.com.br

${ }^{4}$ Tecnóloga em irrigação formada pelo Instituto Federal de Educação, Ciência e Tecnologia - IFCE, campus

Sobral, Ceará. Estudante de Doutorado em Solos e Nutrição de Plantas. Departamento de Solos $\quad \square /$ UFC.

Fortaleza- CE. Brasil. E-mail: nayararochelli@hotmail.com

$\square / \mathrm{UFC}$. 


\section{ABSTRACT}

The basin of Curu Valley in the state of Ceara has a considerable importance in water supply for domestic consumption, industry, fishing activities and mainly for irrigation of agricultural areas. The Curu River, main River of Basin receives polluted water from various (several) places, including communities, shrimp ponds, and others. Which can salt the source of water placing agricultural production in the region of risk, therefore, the objective of this study was to evaluate the quality of water throughout the river route, taking into account the presence of salts. It was chosen a dry period, 21 collection points from the source to the mouth of the river. In all water samples were analyzed electrical conductivity (EC), $\mathrm{pH}$, calcium $\left(\mathrm{Ca}^{2+}\right)$, magnesium $\left(\mathrm{Mg}^{2+}\right)$, potassium $\left(\mathrm{K}^{+}\right)$, sodium $\left(\mathrm{Na}^{+}\right)$, chloride $\left(\mathrm{Cl}^{-}\right)$, bicarbonate $\left(\mathrm{HCO}^{3-}\right)$, and sulfate $\left(\mathrm{SO}_{4}{ }^{2-}\right)$. Regarding the presence of salts, in most of the Curu River, water meet with moderate restrictions for the use of irrigation In mangroves and river mouth, the salt concentration was quite high, but near these points there is no presence of arable land that they can use these waters. It was concluded that the quality of water has moderate restriction for irrigation in most of its length, with the exception of points in the mangroves and the mouth of the river, that the influence of the tide, has high amounts of salts..

Keywords: Hydrographic Basin. Irrigation. Pollution.

\section{INTRODUÇÃO}

A água como fator ambiental é essencial à vida. É um elemento necessário a diversas atividades humanas e constitui componente indispensável no meio ambiente (VASCONCELOS et al., 2009). É considerada essencial na agricultura, pois a sua falta pode provocar vários prejuízos econômicos na produção agrícola e queda na produção de alimentos (NAKAI et al., 2013).

A qualidade da água é um aspecto fundamental para considerar seu uso. Este uso tem sofrido restrições significativas devido à poluição dos rios e outras fontes, provenientes das ações naturais e antrópicos, as quais alteram os aspectos de qualidade e, dessa forma, a quantidade de água de boa qualidade disponível para ser utilizada nas atividades humanas (SOUSA et al., 2014).

Os principais problemas avaliados na água para a irrigação são relacionados à salinidade e a toxicidade por íons (SILVA et al., 2011). A utilização de águas salinas na irrigação pode comprometer as propriedades físicas, químicas e biológicas do solo, afetar negativamente o crescimento e desenvolvimento das plantas e diminuir a produção; portanto, a agricultura irrigada depende da qualidade da água, que, geralmente, é avaliada pela composição individual dos íons presentes (VELOSO et al., 2012)

A Bacia Hidrográfica do Vale do Curu é uma das regiões agrícolas mais importantes do Estado do Ceará, cuja produção se baseia na irrigação. Contudo, apresenta problemas que colocam em risco a qualidade da água do rio principal, como a proximidade de viveiros de camarão, curtumes, indústrias, lixões, matadouros, esgotos doméstico e hospitalar, entre outras fontes de contaminação (ANJOS, 2013).

O rio Curu, principal rio da Bacia do Vale do Curu, nasce nas regiões montanhosas localizadas no centro-norte do Estado do Ceará, percorrendo 195 km de extensão, desde a nascente até a sua foz. Possui $8.605 \mathrm{Km}^{2}$ de área (GORAYEB et al., 2005), representando aproximadamente $6 \%$ do território cearense (INESP, 2009) e abastece 15 municípios: Itatira, Canindé, Caridade, Paramoti, General Sampaio, Tejuçuoca, Apuiarés, Pentecoste, Itapajé, Irauçuba, Umirim, São Luís do Curu, São Gonçalo do Amarante, Paraipaba e Paracuru (GORAYEB et al., 2005). Os seus principais afluentes são o rio Canindé, que se encontra na margem direita e o rio Caxitoré localizado na margem esquerda. 
Na região há a produção agrícola dos perímetros irrigados com o cultivo de coco e banana, e em pequenas propriedades rurais com o cultivo de milho, feijão, e com a criação de pequenos animais como suínos, bovinos, caprinos e aves (COGERH, 2014).

Apesar da importância econômica, social e ambiental da Bacia do Vale do Curu para um expressivo número de habitantes, são poucas as informações referentes às condições atuais da qualidade das águas dos mananciais. Diante disso, este trabalho teve como objetivo avaliar a qualidade da água para uso na irrigação em todo o percurso do Rio Curu, desde a nascente até a foz, no período de estiagem, levando em consideração parâmetros que podem quantificar a salinidade das águas.

\section{MATERIAIS E MÉTODOS}

A área de estudo foi ao longo do percurso do Rio Curu, desde a nascente até a foz, totalizando 21 pontos de amostragem. A coleta das amostras de água foi realizada no período seco, nos meses de julho e agosto de 2014. Na seleção dos pontos, levaram-se em consideração locais onde existiam possíveis fontes de poluição despejadas na água.

Utilizaram-se frascos de polietileno (500 $\mathrm{ml}$ ), previamente lavados com água dos respectivos locais de amostragem. A coleta foi feita aproximadamente a $30 \mathrm{~cm}$ da superfície do rio, contra a corrente. Terminada a amostragem, os frascos foram identificados e mantidos em caixa de isopor, após isso, foi efetuado o transporte até o laboratório, onde foram refrigeradas até o momento de realização das análises. Os locais de amostragem e suas respectivas coordenadas são apresentados na Tabela 1.

Tabela 1. Coordenadas e Referências dos 21 pontos de amostragem de água coletados ao longo do Rio Curu.

\begin{tabular}{|c|c|c|}
\hline Pontos & Coordenadas & Referências \\
\hline 1 & 042॰47’73’’W - 9497'519’'S & Nascente do Rio \\
\hline 2 & $042^{\circ} 40^{\prime} 84^{\prime \prime} \mathrm{W}-94^{\circ} 97^{\prime} 896^{\prime \prime} \mathrm{S}$ & Cacimba de alvenaria no leito do rio \\
\hline 3 & 04495'52'’W - 9551'154'’s & Balneário Recreativo I \\
\hline 4 & $045^{\circ} 06^{\prime} 02^{\prime \prime} \mathrm{W}-95^{\circ} 52^{\prime} 785^{\prime \prime} \mathrm{S}$ & Curtume \\
\hline 5 & $045^{\circ} 23$ '85'’W - 9563’443'’s & Amontante da cidade Apuiarés \\
\hline 6 & 046¹4'36’’W - 95²'’562'’s & Balneário recreativo II \\
\hline 7 & $046^{\circ} 26^{\prime} 23^{\prime \prime \prime} \mathrm{W}-95^{\circ} 74^{\prime} 216^{\prime \prime} \mathrm{s}$ & Próximo ao Açude I \\
\hline 8 & 04708'71'’W - 9580’307'’s & Próximo ao Açude II \\
\hline 9 & 0466'31'’W - 95'80’363'’s & Perímetro Irrigado I \\
\hline 10 & 04694'19’’W - 9582'514’'S & Proximidade de Lixão \\
\hline 11 & 04689'98'’W - 95'86'965', S & Produção de grama sintética \\
\hline 12 & 046'95'14'’W - 95'89'147'’s & Encontro dos rios Caxitoré e Curu \\
\hline 13 & 047³0’16’’W - 95'93’865’'s & Amontante da cidade de São Luis do Curu \\
\hline 14 & 04768’24’’W - 9602’833'’s & Ajusante da cidade de São Luis do Curu \\
\hline 15 & $048^{\circ} 12^{\prime} 44^{\prime \prime} \mathrm{W}-96^{\circ} 10^{\prime} 098^{\prime \prime} \mathrm{S}$ & Comunidade I \\
\hline 16 & $048^{\circ} 30^{\prime} 45^{\prime \prime} \mathrm{W}-96^{\circ} 14^{\prime} 004^{\prime \prime} \mathrm{S}$ & Estação de irrigação \\
\hline 17 & $048^{\circ} 47^{\prime} 12^{\prime \prime \prime} \mathrm{W}-96^{\circ} 15^{\prime} 373^{\prime \prime} \mathrm{s}$ & Perímetro irrigado II \\
\hline 18 & 04863'81'’W - 96²19'613'’s & Comunidade Poço Doce \\
\hline 19 & 048०80'19’'W - 96²1'787'’s & Comunidade II \\
\hline 20 & 049॰10'62'’W - 96²2'663'’s & Mangue \\
\hline 21 & 049³8'87’’W - 96²2'884'’s & Foz do rio \\
\hline
\end{tabular}

As análises foram realizadas no Laboratório de Química e Fertilidade do Solo, do Departamento de Solos da Universidade Federal do Ceará. Foram realizadas análises de $\mathrm{pH}$, condutividade elétrica (CE), dos íons cálcio $\left(\mathrm{Ca}^{2+}\right)$, magnésio $\left(\mathrm{Mg}^{2+}\right)$, potássio $\left(\mathrm{K}^{+}\right)$, sódio 
$\left(\mathrm{Na}^{+}\right)$cloreto $\left(\mathrm{Cl}^{-}\right)$, bicarbonato $\left(\mathrm{HCO}_{3}{ }^{-}\right)$e sulfato $\left(\mathrm{SO}_{4}{ }^{2-}\right)$. Também foi avaliada a Relação de Adsorção de Sódio (RAS) que indica o percentual de sódio trocável. As técnicas utilizadas foram baseadas nas propostas estabelecidas por Embrapa (1979), MAPA (1994) e a classificação da água interpretada de acordo com Richards (1994). De acordo com a classificação de Richards (1954), as águas são divididas em quatro classes com base nos valores da condutividade elétrica (CE), recebendo denominações de $\mathrm{C}_{1}, \mathrm{C}_{2}$, $\mathrm{C}_{3}$ e $\mathrm{C}_{4}$, e outras quatro classes com base na relação de adsorção de sódio (RAS) recebendo denominações de $S_{1}, S_{2}, S_{3}$ e $S_{4}$.

Após as análises, foram realizadas análises de variância e teste de Tukey para comparações entre as médias dos resultados obtidos para todos os parâmetros avaliados, nos 21 pontos de amostragem, com auxílio do software ASSISTAT, versão 7.7 BETA.

\section{RESULTADOS E DISCUSSÃO}

Nas águas do rio Curu, predominam na maior parte do seu trecho águas do tipo $\mathrm{C}_{2} \mathrm{~S}_{2}$ e $\mathrm{C}_{4} \mathrm{~S}_{1}$, que possuem as seguintes interpretações, segundo Richards (1954):

$\mathrm{C}_{2}$ : Água de média salinidade. Pode ser utilizada sempre e quando houver uma lixiviação moderada de sais.

C4: Água de muita alta salinidade. Não é apropriada para irrigação sob condições normais. $\mathrm{S}_{1}$ : Água de baixa sodicidade ou com baixas concentrações de sódio. Pode ser utilizada para a maioria dos solos, com pouca probabilidade de se atingir níveis perigosos de sódio trocável.

$\mathrm{S}_{2}$ : Água de sodicidade média ou com média concentração de sódio. Em solos argilosos, o sódio dessa classe de água representa um perigo de dispersão com possível redução em sua porosidade.

De acordo com a Tabela 2, pode-se observar que houve diferenças significativas entre os pontos em relação às características analisadas da água do Rio Curu, ao nível de $1 \%$ pelo teste F, para todas as variáveis.

Tabela 2. Resumo da análise de variância das concentrações dos íons analisados e dos valores de CE e pH do Rio Curu-CE.

\begin{tabular}{ccc}
\hline Parâmetros & Quadrado Médio & F calculado \\
\hline $\mathrm{Ca}^{2+}$ & 49.04405 & $15663.1300^{* *}$ \\
$\mathrm{Cl}^{-}$ & 108147.60356 & $00022688308^{* *}$ \\
$\mathrm{~K}^{+}$ & 45.31521 & $2374.7708^{* *}$ \\
$\mathrm{HCO}_{3}$ & 1.44642 & $149.2798^{* *}$ \\
$\mathrm{Mg}^{2+}$ & 2608.72056 & $145820.72^{* *}$ \\
$\mathrm{Na}^{+}$ & 72050.52113 & $16431.0461^{* *}$ \\
$\mathrm{pH}$ & 0.67109 & $45.6618^{* *}$ \\
$\mathrm{CE}$ & 164.43187 & $95094.3593^{* *}$ \\
$\mathrm{SO}_{4}{ }^{2-}$ & 0.47345 & $11791.5364^{* *}$ \\
\hline
\end{tabular}

** = significativo a $1 \%$ de probabilidade pelo teste $\mathrm{F}$.

Os valores das médias das análises de $\mathrm{pH}$, condutividade elétrica (CE) e das concentrações dos cátions cálcio $\left(\mathrm{Ca}^{2+}\right)$, magnésio $\left(\mathrm{Mg}^{2+}\right)$, potássio $\left(\mathrm{K}^{+}\right)$, sódio $\left(\mathrm{Na}^{+}\right)$, dos ânions cloreto $\left(\mathrm{Cl}^{-}\right)$, sulfato $\left(\mathrm{SO}_{4}{ }^{2-}\right)$ e bicarbonato $\left(\mathrm{HCO}_{3}{ }^{-}\right)$são apresentados na Tabela 3.

Tabela 3. Comparação entre as médias de $\mathrm{pH}, \mathrm{CE}$, das concentração dos cátions $\mathrm{Ca}^{2+}, \mathrm{Mg}^{2+}, \mathrm{K}^{+} \mathrm{e} \quad \mathrm{Na}^{+}$, $\mathrm{Cl}^{-}, \mathrm{SO}_{4}{ }^{2-}, \mathrm{HCO}_{3}{ }^{-}$para os 22 pontos de amostragem coletados.

\begin{tabular}{|c|c|c|c|c|c|c|c|c|c|c|}
\hline Pontos & pH & $\mathrm{CE}$ & $\mathbf{C a}^{2+}$ & $\mathbf{M g}^{2+}$ & $\mathbf{K}^{+}$ & $\mathrm{Na}^{+}$ & $\mathrm{Cl}^{-}$ & $\mathrm{HCO}_{3}{ }^{-}$ & $\mathrm{CO}_{3}{ }^{2-}$ & $\mathrm{SO}_{4}{ }^{2-}$ \\
\hline & & dS $\mathrm{m}^{-1}$ & & & & $\mathbf{m m}$ & & & & \\
\hline
\end{tabular}




\begin{tabular}{|c|c|c|c|c|c|c|c|c|c|c|}
\hline $\mathrm{P} 1$ & 7,7 ef & $1,26 \mathrm{e}$ & 0,58 ef & 3,46 e & 0,27 e & 8,32 e & 12,7 e & $0,16 \mathrm{~g}$ & $0,00 \mathrm{~h}$ & 0,11 ef \\
\hline P2 & $0 \mathrm{~cd}$ & 0,51 mno & ii & $1,49 \mathrm{fg}$ & e & $1 \mathrm{e}$ & op & 5 de & $0,64 \mathrm{e}$ & 0 \\
\hline P3 & 5 ef & $79 \mathrm{f}$ & e & $1,76 \mathrm{f}$ & e & 5,25 e & 5,0 & ,94 a & $0,00 \mathrm{~h}$ &, $04 \mathrm{fg}$ \\
\hline $\mathrm{P} 4$ & 7,6 ef & 0,57jlmon & $0,41 \mathrm{fg}$ & $1,70 \mathrm{f}$ & 0,25 e & 3,70 e & 3,4 no & $2,10 \mathrm{bc}$ & $0,00 \mathrm{~h}$ & 0,05 efg \\
\hline P5 & 7,8 de & 0,60 ijlmn & $0,498 \mathrm{f}$ & $1,70 \mathrm{f}$ & 0,32 e & 3,53 e & 4,3 l & $2,3 \mathrm{~b}$ & $0,00 \mathrm{~h}$ & $0,03 \mathrm{fg}$ \\
\hline P6 & 7,5 ef & 0,60 ijlmn & 0,57 ef & $1,63 \mathrm{f}$ & 0,33 e & 3,9 e & 4,2 & $2,24 \mathrm{c}$ & $0,00 \mathrm{~h}$ & $0,05 \mathrm{fg}$ \\
\hline P7 & 7,8 de & 0,60 ijlmn & 0,50 ef & $1,6 \mathrm{f}$ & 0,3 & 4,16 e & $4,20 \mathrm{~m}$ & $2,01 \mathrm{c}$ & $0,00 \mathrm{~h}$ & 0,08 efg \\
\hline P8 & 8 a & 0,67 fghij & $0,12 \mathrm{ij}$ & $1,65 \mathrm{f}$ & 0,23 e & 5,0 e & 5,0 hi & $1,24 \mathrm{e}$ & $0,81 \mathrm{c}$ & $0,04 \mathrm{fg}$ \\
\hline P9 & $8,5 a b$ & $0,74 \mathrm{fg}$ & 0,21hij & $1,5 \mathrm{f}$ & 0,20 e & $0 \mathrm{e}$ & $4,0 \mathrm{~m}$ & $2,9 \mathrm{a}$ & $0,70 \mathrm{~d}$ & efg \\
\hline 10 & $8,5 a b$ & 0,50 no & 0,21hij & $1,15 \mathrm{gh}$ & 0,18 e & 3,5 e & 3,3 no & 1,40 de & $0,90 \mathrm{~b}$ & 0,03 fg \\
\hline 11 & 8,3 bc & 0,53 lmno & 0,58 ef & $1,25 \mathrm{f}$ & $0,42 \mathrm{e}$ & 3,7 e & 3,50 no & $1,48 \mathrm{~d}$ & $1,0 \mathrm{a}$ & $0,03 \mathrm{fg}$ \\
\hline P12 & 8,7 a & 0.60 ijlmn & 0,20hij & $1,5 \mathrm{fg}$ & C & 4,5 e & 4,8 & $0,85 \mathrm{f}$ & $0,80 \mathrm{c}$ & 0,03 fg \\
\hline 13 & $8,5 \mathrm{ab}$ & 0,62 о & $0,079 \mathrm{j}$ & $1,6 \mathrm{f}$ & 0,18 e & 4,0 e & & $0,70 \mathrm{f}$ & $0,61 \mathrm{f}$ & $0,01 \mathrm{~g}$ \\
\hline P14 & $8,2 \mathrm{ab}$ & 0,64 ghijl & 0,22hij & $1,5 \mathrm{fg}$ & 0,20 e & $5,0 \mathrm{e}$ & $4,80 \mathrm{ij}$ & $1,60 \mathrm{~d}$ & $0,51 \mathrm{~g}$ & $0,03 \mathrm{fg}$ \\
\hline 15 & 8,7 a & J,63ghijlm & 0,14hij & 1,42 fg & $0,22 \mathrm{e}$ & 5,1 & $5,0 \mathrm{~h}$ & 1,1 & $0,60 \mathrm{f}$ & 0,03 fg \\
\hline 16 & 7,8 de & 0,73 fgh & 0,21hij & $1,50 \mathrm{fg}$ & 0,24 e & 5,7 e & $6,0 \mathrm{f}$ & 1,30 de & $0,00 \mathrm{~h}$ & 0,05 \\
\hline P17 & $8,6 \mathrm{ab}$ & 0,74 fghi & 0,15hij & $1,53 \mathrm{fg}$ & $0,24 \mathrm{e}$ & 5,7 & $5,8 \mathrm{~g}$ & 1,3 de & $0,68 \mathrm{~d}$ & 0,05 \\
\hline P18 & 7,7def & 0,71 fghi & 0,30 gh & $1,50 \mathrm{fg}$ & 0,25 e & J, & 6 , & 1,3 & $0,00 \mathrm{~h}$ & 0,15 e \\
\hline P19 & 7,8 de & $15,3 \mathrm{c}$ & 6,61 c & 43,36 c & $5,74 \mathrm{c}$ & $225,93 c$ & $277,51 \mathrm{c}$ & $2,01 \mathrm{c}$ & $0,00 \mathrm{~h}$ & 3,46 a \\
\hline P20 & 7,7 ef & $7,1 \mathrm{~b}$ & $56 \mathrm{~d}$ & $17,79 \mathrm{~d}$ & $2,59 \mathrm{~d}$ & $98,05 \mathrm{~d}$ & $117,2 \mathrm{~d}$ & $1,64 \mathrm{~d}$ & $0,00 \mathrm{~h}$ & $1,60 \mathrm{~d}$ \\
\hline P21 & 7,5 ef & $23,06 \mathrm{~b}$ & $12,31 \mathrm{~b}$ & $92,53 \mathrm{~b}$ & $11,28 \mathrm{~b}$ & $435,6 \mathrm{~b}$ & 52 & $0,81 \mathrm{f}$ & $0,00 \mathrm{~h}$ & $2,8 \mathrm{c}$ \\
\hline 22 & $7,4 \mathrm{f}$ & $25,20 \mathrm{a}$ & $14,84 \mathrm{a}$ & $106,1 \mathrm{a}$ & 14,78 a & 589,2 a & 710,2 a & $1,62 \mathrm{~d}$ & $0,00 \mathrm{~h}$ & $3,17 \mathrm{~b}$ \\
\hline
\end{tabular}
Médias seguidas de letras iguais na coluna são estatisticamente iguais, ao nível de 5\% de probabilidade, pelo teste de Turkey.

Os valores de $\mathrm{pH}$ obtidos nas amostras coletadas ao longo do Rio Curu variaram entre 7,4 e 8,8 (Tabela 3). A faixa normal de $\mathrm{pH}$ da água para irrigação varia entre de 6,5 a 8,4. Os principais pontos que ultrapassaram os limites recomendados para irrigação, localizam-se próximos a cidades, indústrias, perímetros irrigados, no encontro do rio Curu com os rios Canindé e Caxitoré.

Geralmente um pH elevado está associado à decomposição da matéria orgânica, poluição e às altas taxas respiratória 
dos microrganismos (MORAES, 2008) e a presença de algas na água. Isto por que, ao realizarem fotossíntese, as algas utilizam o gás carbônico, que é a principal fonte natural de acidez (SILVA et al., 2007).

A condutividade elétrica (CE) da água nos locais de amostragem ao longo do Rio Curu, variaram de 0,50 dS m ${ }^{-1}$ a 23,06 dS m${ }^{-1}$. No entanto, na maior parte do trecho do Rio a CE variou de 0,50 a $0,79 \mathrm{dS} \mathrm{m}^{-1}$. Estes valores se encontram muito próximo do limite recomendado para a irrigação, que varia de 0 a $0,7 \mathrm{dS} \mathrm{m}^{-1}$, apresentando restrição moderada para irrigação. $O$ maior valor foi encontrado na foz do rio $\left(23,06 \mathrm{dS} \mathrm{m}^{-1}\right)$, devido à proximidade do mar que influenciou no aumento da concentração de sais da água.

A concentração do $\mathrm{K}^{+}$nas águas analisadas variou de 0,148 a $11,28 \mathrm{mmol}_{\mathrm{C}} \mathrm{L}^{-1}$. Contudo, na maior parte do Rio Curu a concentração do íon potássio está de acordo com valores encontrados em águas superficiais, não apresentando problemas para irrigação segundo a classificação da University of California Committee of Consulants (PIZARRO, 1985).

A concentração de $\mathrm{Ca}^{2+}$ na água da maior parte do trajeto do Rio Curu variou de 0,079 a 0,68 $\mathrm{mmol}_{\mathrm{c}} \mathrm{L}^{-1}$. A concentração do íon magnésio $\left(\mathrm{Mg}^{2+}\right)$ na água, assim como $\mathrm{o}$ cálcio, na maioria dos pontos coletados variou de 1,15 a 3,4 mmol $_{\mathrm{c}} \mathrm{L}^{-1}$. Sendo este último valor encontrado na nascente do rio. Os maiores valores para estes íons foram encontrado na foz do rio. A disponibilidade de cálcio entre os ambientes marinhos, terrestres e de água doce é bastante variável. No ambiente marinho, o íon é o quarto mais abundante sendo bem mais elevado que no ambiente de água doce (MAIER et al., 2011). O magnésio é o terceiro elemento mais abundante encontrado nas águas do mar, ficando atrás apenas do sódio e do cloro (NAKURAMA;MOREJON, 2013), explicação para as altas concentrações dos elementos próximo a foz do rio.

$\mathrm{O} \mathrm{Na}{ }^{+}$nas águas coletadas variou na maior parte do rio entre 2,51 a $8,32 \mathrm{mmol}_{\mathrm{c}} \mathrm{L}^{-1}$, sendo esta última encontrada na nascente do rio, que devido ao período seco, se encontrava concentrada no ponto de coleta, explicação para a maior quantidade de sais na mesma. Contudo, todos estão dentro da faixa normal de concentração do elemento para a irrigação (0 $\left.9 \mathrm{mmol}_{\mathrm{C}} \mathrm{L}^{-1}\right)$; já para irrigação por aspersão, o elemento passa a ter ligeira restrição acima de $3 \mathrm{mmol}_{\mathrm{c}} \mathrm{L}^{-1}$. A maior concentração (435,6 mmolc $L^{-1}$ ) foi encontrada na foz no rio devido à influência da maré. $\mathrm{O} \mathrm{Na}{ }^{+}$em concentrações elevadas no solo pode causar problemas na estrutura do mesmo, como na diminuição da permeabilidade ao ar e à água que interferem diretamente na produção das culturas (FREIRE et al., 2014). Segundo Amaro Filho et al., (2008) os solos saturados com sódio ficam mais hidratados, se dispersam e intumescem, tornando-se impermeáveis, dificultando o desenvolvimento das plantas.

A variação do $\mathrm{Cl}^{-}$, na maior parte da extensão do Rio Curu, foi de 3,3 a 12,70 $\mathrm{mmol}_{\mathrm{c}}$ $\mathrm{L}^{-1}$, sendo este último encontrado na nascente do rio. O valor da concentração de cloreto recomendada para as águas de irrigação por superfície é de 0 - $10 \mathrm{mmol}_{\mathrm{c}} \mathrm{L}^{-1}$, e para irrigação por aspersão, o elemento passa a ter ligeira restrição acima de $3 \mathrm{mmol}_{\mathrm{c}} \mathrm{L}^{-1}$. Por tanto, com exceção dos pontos P1, P19 e P20 e P21, todos os outros se encontram dentro da faixa recomendada para irrigação, sendo, neste caso, a irrigação por superfície a mais indicada devido à concentração de cloretos na água do manancial. Os íons $\mathrm{Na}^{+}$e $\mathrm{Cl}^{-}$no substrato podem reduzir o crescimento das plantas, em virtude destes elementos causarem diversos efeitos negativos, influenciando a capacidade das plantas em absorver, transportar e utilizar alguns dos nutrientes (CRUZ et al., 2006).

Em relação ao bicarbonato, a variação da concentração nas águas do rio foi de 0,16 a 2,94 $\mathrm{mmol}_{\mathrm{C}} \mathrm{L}^{-1}$. A menor concentração do íon $\left(0,16 \mathrm{mmol}_{\mathrm{C}} \mathrm{L}^{-1}\right)$ foi encontrada na nascente do rio e a maior concentração $\left(2,94 \mathrm{mmol}_{\mathrm{C}} \mathrm{L}^{-1}\right)$ foi encontrada no Balneário recreativo. Segundo a classificação da University of California Committee of Consulants (1974) a faixa de concentração de bicarbonato recomendada para irrigação por aspersão convencional de nenhuma restrição à restrição moderada é de 0 - 8,5 mmol $_{\mathrm{c}} \mathrm{L}^{-1}$. Por tanto, todos os pontos ao longo do rio possuem concentrações inferiores 
ao valor limite recomendados, mostrando não possuem restrição para uso na irrigação.

Em relação ao sulfato, a variação da concentração nas águas do rio foi de 0,01 a 3,46 mmol $\mathrm{L}^{-1}$. Os pontos de coletas com as maiores concentrações foram encontrados próximos a foz do rio. A menor concentração $\left(0,01 \mathrm{mmol}_{\mathrm{c}} \mathrm{L}^{-1}\right)$ foi encontrada à montante da cidade de São Luiz do Curu e a maior, próximo a Comunidade II (P19). A baixa concentração de sulfato nas águas pode ser resultado do efeito da diluição do elemento na água e, também, pela redução a sulfetos que ocorre, principalmente, em locais anaeróbicos e ricos em material orgânico (CETESB, 2009).

\section{CONCLUSÃO}

Conclui-se que, em termos qualitativos, a água do Rio Curu, levando-se em consideração os parâmetros estudados, mesmo no período de estiagem, tem moderada restrição para irrigação, devendo-se ser utilizada com cautela, evitando solos argilosos e culturas sensíveis a salinidade. Nos pontos próximos à foz e ao mangue há alta quantidade de sais pela influência da maré, tendo esta água utilização indevida para irrigação.

\section{REFERÊNCIAS}

AMARO, FILHO, J.; JÚNIOR ASSIS, R.N.; MOTA, J.C.A.; Física dos Solo: Conceitos e Aplicações. Fortaleza 2008. Editora Universitária. 115p.

ANJOS, D.C. Levantamento da diversidade microbiana de solos afetados por metais pesados ao longo do Rio Curu. (Projeto de Tese). UFC, 2013.

CETESB, Companhia de Tecnologia de Saneamento Ambiental Significado ambiental e sanitário das variáveis de qualidade das águas e dos sedimentos e metodologias analíticas de amostragem. 2009
COGERH. COMPANHIA DE GESTÃO DOS RECURSOS HÍDRICOS. Bacia Hidrográfica do Curu: Características Gerais. In: http://www.cogerh.com.br/ Acessado em 05 / 08 / 2014.

CRUZ, L.J.; PELACANI, C.R.; COELHO, E.F.; CALDAS, R.; ALMEIDA, A.;QUEIROZ, J.R.; Influência da Salinidade sobre o Crescimento, Absorção e Distribuição de Sódio, Cloro e Macronutrientes em Plântulas de Maracujazeiro-Amarelo. Bragantia, Campinas, v.65, n.2, p.275-284, 2006

EMBRAPA - SNLCS. Manual de métodos de análises de solo. Rio de Janeiro: Serviçõ Nacional de levantamento e Conservação de Solos, 1979.

Freire , M B. G. S. ; Miranda, M. F. A.; Oliveira, E. E. M.; Silva, L E. da,; Pessoa, L. G. M.; Almeida, B G. de; Agrupamento de solos quanto à salinidade no Perímetro Irrigado de Custódia em função do tempo. R. Bras. Eng. Agríc. Ambiental, v.18, (Suplemento), p.S86-S91, 2014.

GORAYEB, A.; SOUZA, M. J. N.; FIGUEIREDO, M. C. B.; ARAÚJO, L. F. P.; ROSA, M. F.; SILVA, E. D. Aspecto geoambientais, condições de uso e ocupação do solo e níveis de desmatamento da bacia hidrográfica do Rio Curu, Ceará - Brasil. UniversidadeEstadual de Londrina, v. 14, n. 2. 2005.

INESP - INSTITUTO DE ESTUDOS E PESQUISAS PARA O DESENCOLVIMENTO DO ESTADO DO CEARÁ. Caderno regional da bacia do Curu. v. 4. Fortaleza /CE. 2009.

MAIER, H. L. Níveis iônicos durante o desenvolvimento embrionário de caranguejos (crustácea) com diferentes graus de terrestrialidade. In: VII Jornada de Iniciação Científica, 2011. XIV COLACMAR. Universidade Presbiteriana Mackenzie, 2011. 
MAPA. Métodos Oficiales de Análisis. Madrid. Ministerio de Agricultura Pesca y Alimentacíon - Mapa, Secretaria General Técnica, 1994.

MORAES, P. B.; Tratamento Biológico de Efluentes Líquidos e Tratamento FísicoQuímico de Efluentes Líquidos. Notas de aula. Curso Superior de Tecnologia em Saneamento Ambiental CESET/UNICAMP

NAKAI, E. H.; ROSA, H. A.; MOREIRA, C.R.;SANTOS, R. F. Qualidade da água utilizada em irrigação no rio São Francisco falso braço Sul- Estado do Paraná. Cultivando o saber. Cascavel, v. 6, n. 4, p. 214 - 224, 2013.

NAKURAMA, A. F. A. N.; MOREJON, C. F. M. Avaliação técnica e econômica de dois processos de produção de magnésio. III Encontro Paranaense de Engenharia e Ciência. Toledo, 2013.

PIZARRO,F.; Drenaje agrícola y recuperacion de suelos salino. 2ed. Madrid. Editorial Espanola S.A, 1985. 542p.

RICHARDS, L. A. Diagnóstico y recuperación de suelos salinos y sódicos. Laboratorio de Salinidad. Ed. México: Editorial Limusa, 1994.

SILVA, D. F.; SOUSA, F. A. S.; KAYANO, M. T. Avaliação dos impactos da poluição nos recursos hídricos da bacia do Rio Mundaú (AL E PE). Revista de Geografia. Recife: UFPE - DCG/NAPA, v. 24, no 3, 2007.

SILVA, I. N.; FONTES, L. O.; TAVELLA, L. B.; OLIVEIRA, J. B.; OLIVEIRA, A. C. Qualidade de Água na Irrigação. ACSA Agropecuária Científica no Semi-Árido, v.07, n 03, 2011.

SOUZA, J.R.; MORAES, M.E.B.; SONODA, S.L.;; SANTOS, H.R.G; A importancia da qualidade da água e os seus mútiplos usos: Caso Rio Almada, Sul da Bahia, Brasil. REDE Revista Eletrônica do Prodema, v.8, n.1, p. 26-45, abr. 2014, Fortaleza, Brasil.

VASCONCELOS, R. S.; LEITE, K. N.; CARVALHO, C. M.;ELOI, W. M.; SILVA, L. M. F; FEITOSA, H. O. Qualidade da água utilizada para irrigação na extensão da microbacia do Baixo Acaraú. Revista Brasileira de Agricultura Irrigada v.3, n.1, p.30-38, 2009.

VELOSO, M. E. C; MOUSINHO, F. E. P.; BLANCO, F. F.; ROCHA JUNIOR, A. F.; BORGES NETO A.; Avaliação e monitoramento da qualidade de água do distrito de irrigação dos tabuleiros de São Bernardo, Maranhão. INOVAGRI. IV Workshop Internacional de Inovações Tecnológicas na Irrigação. Fortaleza - Ce. 2012. 